\title{
EFFECT OF MOULDING SAND’S THERMO-PHYSICAL PROPERTIES ON THE CRYSTALLIZATION OF DUCTILE CAST IRON
}

\author{
Marcela Pokusová \\ Faculty of Mechanical Engineering, Slovak University of Technology in Bratislava \\ e-mail: marcela.pokusova@stuba.sk
}

Keywords: ductile cast iron, chemical composition, thermo-physical properties, moulding sand

\begin{abstract}
In order to obtain good mechanical properties of ductile cast iron, it is necessary that the highest quantity of the regular graphite spheroids are distributed uniformly in a matrix. The paper presents the method that employs the specific criterial quantity - casting modulus for calculating the carbon equivalent, total carbon content and effective wall thickness of a casting. The effective wall thickness is also adjusted by the correction coefficient $\chi$ expressing the influence of the thermo-physical properties of the moulding sand on the solidification time.
\end{abstract}

\section{INTRODUCTION}

The present range of ferrous based materials used for castings the volume of special grades of cast irons increases, when the production of the steel castings decreases permanently. From a long-term point of view, the ductile cast iron, known as the spheroidal graphite iron (SGI), shows sustained steady growth of this production. The graphite takes the shape of nodules due to the conditions of solidification produced by the active elements introduced into the molten metal during its metallurgical treatment. The basic nodulizing element $\mathrm{Mg}$ added in amounts from $0.03-$ $0.06 \%$ with traces of $\mathrm{Ce}, \mathrm{Ca}$ etc... causes the tendency of cast iron to solidify metastably, and therefore, the inoculation has to be done simultaneously or as soon as possible. The aim of inoculation is to form the graphite's nuclei of the supercritical size and promote their growth. To attain good mechanical properties of individual grades of cast iron it is necessary to give increase the regular nodules of the graphite as much as possible and distribute them uniformly throughout the matrix. This depends not only on the chemical composition of the molten metal, but also on the cooling gradients given by the casting section's thickness and the heat conductivity of the mould.

\section{DETERMINATION OF THE CHEMICAL COMPOSITION OF DUCTILE CAST IRON}

The effect of the casting's section on the structure and mechanical properties of nonalloyed SGI was examined on casting samples with a step-plate section. The thicknesses of steps were $3,6,12,24,48$, and $96 \mathrm{~mm}$, and their width and length were always six times the corresponding thickness. The meltings were performed in an induction furnaces with the crucible of 100 or $500 \mathrm{~kg}$ using the charge based on basic pig iron having an S content of $0.016-0.020 \%$. The materials of the individual melting had an equivalent carbon content $(C E)$ of 3.9 to $5 \%$, when the $\mathrm{C}$ content was in the range of 3 to $4.2 \%$ and the $\mathrm{Si}$ content from 1.9 to $3.5 \%$. To ensure the reproducibility of the results for the metallurgical processing of molten iron, the base iron was poured simultaneously over the nodulizer and the inoculant in the ladle was used and, for comparison, we apply the in-mould technology. For treatment of the molten iron, the modifier of 
FeSiMg7 of commercial quality was used in both methods. Apart from the study of mechanical properties, the main criterion was to obtain the structure having the graphite in the shape of regular nodules uniformly distributed in the matrix. Furthermore, the formation of predominantly pearlitic matrix occurred, found throughout the boundary in the development of hard spots, the occurrence of the defect graphite, and size of shrinkage. The experimental results enabled show the dependence between $C E$ and the wall thickness, at which the material of the casting has the highest ductility, strength ranged from 500 to $600 \mathrm{MPa}$ [1], and the boundaries of hard spots, defected or improper distributed graphite nodules.

Statistical processing of the results indicated a correlation between the thickness of the section and the contents of the total and equivalent carbon, at which the highest ductility and notch toughness are achieved, including the highest nodularity of regular nodules of graphite. The regression analysis of the attained dependences allowed for the construction of semi-empirical equations for the direct calculation of $C E$ and total carbon content $C$ from formulas, which are valid for green moulding in bentonite sands:

$$
\begin{aligned}
& C E=3,18-0,32 \log 2 \\
& C=4,8=0,46 \log ^{2}
\end{aligned}
$$

In Eq. (1) and (2) the wall thickness of the casting $t$ is in mm and the calculated values of $C E$ and $C$ are in wt. \%. Equations are valid for the sections from 3 to $100 \mathrm{~mm}$. The content of silicon can be derived from the known expression [1] used for calculating the $C E$ :

$$
C E=C+0,315 t \quad \text { or } \quad S t=3.224 C E-C)
$$

Eq. (3) omits other common minor elements, because their content is usually very low in non-alloyed SGI, and their effect on $C E$ is negligible. In the given case, the content of phosphorus was about $0.05 \%$, content of manganese about $0.4 \%$, and the presence of other carbide promotive elements were strictly limited. From the technological viewpoint, no doubt the content of the residual magnesium is very important. According to [1, 2, 3], in thin sections, the content of $\mathrm{Mg}$ should be closer to the lower boundary, which reduces the demands on inoculation. For heavy sections, it is necessary to apply higher levels of nodularization with an $\mathrm{Mg}$ content closer to the upper boundary, which naturally increases the intricacy of inoculation. The reasoning for this attitude was proved from the results of the melting processes, where the $\mathrm{Mg}$ content was selected in the range of $0.04-0.07 \mathrm{wt} . \%$.

\section{EFFECT OF THE CASTING'S SHAPE AND THERMO-PHYSICAL PROPERTIES OF SAND MOULDING ON THE CRYSTALLIZATION OF DUCTILE CAST IRON}

The most common method applied for pouring into green bentonite sand moulds, the properties of SGI depend, apart from the pouring method, also on the castings wall section and the procedure of metallurgical treatment of the molten metal. The solidification rate is specified by the thickness of the metal layer $x$ solidified on the mould's wall, which is directly proportional to the square root of solidification time $\tau$ expressed in the equation:

$$
x=k \sqrt{i}
$$


where $k$ is the coefficient of solidification allowing for the balance between the quantity of heat liberated from the casting transferred into the mould.

Eq. (4) expresses the parabolic dependence of the thickness of the solidified layer on time, when (at time $\tau_{0}$ ) the final state of solidification of an infinitely large plate having a thickness of $t$ both isosolidi meet in the centre of the plate, where $x=\frac{t}{2}$. The given dependence are valid for castings formed by solids, which have a length and width which are at least six times larger than their thickness. At different figures the volume to cooled area ratio changes, which results in changing of cooling rate and also the thermo-dynamic conditions of crystallization. It is known that the castings with various shapes solidify at the same rate, if the values of their volume $V$ to their area $A$ ratio are tallied, i.e. the value of a modulus $M$ is $M=\frac{V}{A}$. In an infinitely large plate, the thickness of the solidified layer $x=\frac{v}{2}$ is numerically equal to the ratio $\frac{V}{A}$, and we have:

$$
M=\frac{V}{d}=\frac{2}{2}=\sqrt{2} \sqrt{r_{0}} \quad \text { or } \quad \nabla_{0}=\frac{M^{2}}{k^{2}}
$$

From Eq. (4) and (5), besides the modulus $M$ the coefficient $k$ has an effect on the solidification time $\tau$, which controls the course of solidification. Prior to introducing the computer simulation, for the particular cases, the values of $k$ were determined experimentally. The accurate computation of the coefficient $k$ can be done by solving the differential equations for heat transfer and conduction in the casting and mould using the finite element method. This approach is utilized by software for the simulation of the solidification processes.

From Eq. (5) it follows that in Eq. (1) and (2) the wall thickness $t$ can be substituted with the modulus $M=\frac{c}{2}$. For example, the solid cylinder with the diameter $d$ has the modulus $M=\frac{a}{4}$, the square rod with the side $b$ has $M=\frac{b}{4}$, and the cube with the length of edge $a$ has $M I=\frac{a}{b}$. If we substitute the thickness $t$ with the modulus $N=\frac{t}{2}$ in Eq. (1) and (2) the equations take the form:

$$
\begin{aligned}
& C E=4,97-0,32 \log V t \\
& C=4.16=0.46 \log B 8
\end{aligned}
$$

If there are walls with different thickness or with parts of different moduli $M$ in the casting, our experiments has shown that in order to keep the acceptable ratio between the content of ferrite and pearlite in the slowest and quickest solidifying parts, it is best to select the arithmetic average of $C E$ and $C$ contents computed for the boundary values of the moduli $M_{\max }$ and $M_{\min }$ of the critical casting's parts [3] according to Eq. (6) and (7):

$$
C E=4.97-0.26 \log \left(M_{\operatorname{man}} n_{\min }\right)
$$




$$
C=4.16-0.23 \log \left(N_{\operatorname{mas}} x_{\min }\right)
$$

Eq. (8) and (9) take into consideration that the parts solidifying under different rates can occur in the actual castings, and ensure that the acceptable difference in the structure and the mechanical properties will be kept in the parts with the highest and lowest value of the modulus. Castings having a modulus below $5 \mathrm{~mm}$ (thickness $\leq 10 \mathrm{~mm}$ ) the ratio of the modulus boundary values can be $H_{\max }: U_{\min }<0: 1$. Mainly in heavy sectioned castings, with a modulus more than $10 \mathrm{~mm}$, the ratio of $4: 1$ causes too great of a difference in the structure and material properties. If it is possible, walls thicker than $50 \mathrm{~mm}$ or those having a modulus above $25 \mathrm{~mm}$ should be avoided. Generally, in the heavy-sectioned castings it is problematic to achieve nearly optimal structural and mechanical properties due to the very close interval of usable chemical composition. For castings poured from SGI, thin sections (below $10 \mathrm{~mm}$ ) are favourable because in thinner walls the material always attains a higher strength and also, more importantly, obtain higher values of ductility and toughness compared to thicker walls. In manufacturing of SGI, some deviations of the chemical composition from those determined according to Eq. (1) to (3) and (6) are tolerable, and we can eliminate them by applying more effective inoculation e. g. by a multiple one.

Most production with castings made from graphitic irons, including SGI, are poured into green sand moulds made from moulding sands, where the basic components are silica and bentonite as the binding material. This technology ensures low production costs and satisfactory surface quality and the existence of the condensation zone [3] which eliminates the implications of the dilations caused by non-uniform cooling of the casting, especially by pre-shrinkage expansion. If there are increased requirements on the surface quality or the casting's accuracy the producers apply second generation moulding sands based on a synthetic resin binding system, which make shaking-out the castings from the mould easier. However, they introduce a new factor in the solidification process that changes the thermo-physical conditions of the solidification.

From literature, many relations exist on the determination of coefficient $k$. These equations are mostly valid for the solidification of rectangular solids or long cylinders, thus the geometrical solids characterized by the linear or radial direction of heat exchange. In [3], the formula for calculating the coefficient $k$ according to Prribil is quoted:

$$
\hat{n}=\frac{\sqrt{\pi}\left(\tau_{s}-\tau_{f}\right) b_{p}}{2 \rho\left[c\left(T_{l}-\tau_{s}\right)+\pi\right.}
$$

where, $T_{s}$ is the temperature of solidus ( $T_{s}=1423 \mathrm{~K}$ for SGI), $T_{f}$ is the starting temperature of mould (K), $\rho$ is the density of molten metal $\left(\rho=7.10^{3} \mathrm{~kg} \mathrm{~m}^{-3}\right.$ for SGI), $c$ is the specific heat of melt $\left(c=540 \mathrm{~J} \mathrm{~kg}^{-1} \mathrm{~K}^{-1}\right.$ for SGI), $T_{l}$ is the temperature of the pour $(\mathrm{K}), l$ is the heat of solidification $\left(l=230.10^{3} \mathrm{~J} \mathrm{~kg}^{-1}\right.$ for SGI), $b_{f}$ is the coefficient of heat accumulated in the mould $\left(\mathrm{Ws}^{-1 / 2} \mathrm{~m}^{-2} \mathrm{~K}^{-1}\right)$, when $b_{f}=\sqrt{\lambda_{f} c_{f} \rho_{f}}$ the physical properties of the moulding sald, such as its heat conductivity $\lambda_{f}$ density $\rho_{f}$. 
If we assume that the properties of the molten metal, like the intensity of overheating and its physical properties, are constant then the change of the moulding technology can only impact the coefficient of heat accumulation in the mould. The experiments focused on comparing green moulds made from bentonite moulding sand containing about $3.5 \%$ of water with moulds made from the resin moulding sand. This combination of the moulding sands is selected because they are mostly used for pouring SGI and can give good reproducibility of the results, which can be promptly implemented in practice. During experiments, the coefficient $k$ was determined experimentally, by measuring with thermocouples, in order to compare the recorded course of solidification with the computed values, the simulation software WinCast is used. After statistical processing of the results obtained from experiments, the value of coefficient $k$ was determined for the given bentonite moulding sand and the casting of shape like cylinder or plate, where $k=0.94$ $\mathrm{mm} . \mathrm{s}^{-1 / 2}$ or $k=0.0564 \mathrm{~m} \cdot \mathrm{h}^{-1 / 2}$. Inserting the values of the physical properties of SGI molten metal poured under overheating of $200 \mathrm{~K}$, the coefficient of heat accumulation of the mould made from the bentonite moulding sand $b_{f(b)}=1587 \mathrm{Ws}^{-1 / 2} \mathrm{~m}^{-2} \mathrm{~K}^{-1}$ is calculated. Solving Eq. (5) and (10), the correction factor $\chi$ is derived, adjusting the thickness of the casting's wall $t$ against the modulus:

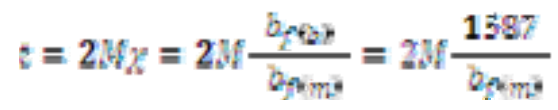

When the moulding sands with water glass are used, the conditions of cooling of the castings do not change, because their typical value of $b_{f}=1510 \mathrm{Ws}^{-1 / 2} \mathrm{~m}^{-2} \mathrm{~K}^{-1}$ is virtually equal to those of the bentonite sands. Introducing the moulding sands based on the foundry silica sand of S22 bonded with the phenol-formaldehyde resin [4], the conditions of cooling have changed substantially therefore this moulding sand has the value of product $\rho_{f} \cdot c_{f}=1.4 \mathrm{MJ} \cdot \mathrm{m}^{-3} \mathrm{~K}^{-1}$, heat conductivity of $\lambda_{f}=0.8 \mathrm{~W} \cdot \mathrm{m}^{-1} \mathrm{~K}^{-1}$, and the value of $b_{f}=1058 \mathrm{Ws}^{-1 / 2} \mathrm{~m}^{-2} \mathrm{~K}^{-1}$. According to Eq. (11) the value of the correction factor $\chi$ is $\chi \approx 1.5$ and the value of the wall thickness is $t=3 \mathrm{~mm}$. When the wall thickness is $2 \mathrm{~mm}$, i.e. modulus of $1 \mathrm{~mm}$, the casting will solidify at an equal rate to casting having a thickness of $3 \mathrm{~mm}$.

When pouring heavy sectioned castings into water-free moulding sands with synthetic resin binder, it is desirable to allow for reduced heat transfer. Assuming for equal overheating of the molten metal and the identical casting, when we substitute the bentonite moulding sand for the water-free resin sand, only the value of the coefficient of heat accumulation of the mould changes from $b_{f(b)}$ to $b_{f(m)}$. Using Eq. (4), (5) and (10) we can calculate the corresponding change of the casting's solidification time in the bentonite sand from $\tau_{0(b)}$ to $\tau_{0(m)}$ and in the resin moulding sand:

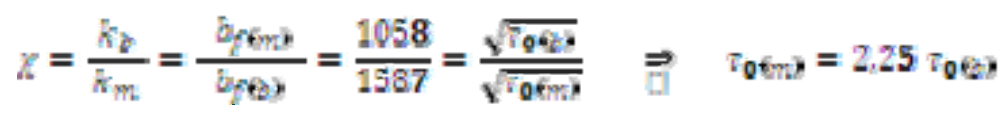

On Fig. 1, the results from the computer simulation of solidification for the industrial casting of the stand poured from ductile cast iron of grade STN 422314 are presented. The casting with the weight of $25 \mathrm{~kg}$ was commonly poured at the faculty's foundry laboratory. Simulation of the solidification process was performed in conditions of the bentonite moulding sand and the phenol-formaldehyde bonded moulding sand system. The casting was formed from conical part 
with the base having a thickness of about $20 \mathrm{~mm}$ and the bearing tube of the thickness $30 \mathrm{~mm}$. Fig. 1a documents the time course of the casting solidification in the bentonite mould, where after pouring the thin walled part solidified during 123 to 400 seconds and the cylindrical part within 400 to 680 seconds. The solidification finished completely within 700 seconds after pouring, when the isosolidi closed in the hot spot, i.e. where the conical and cylindrical parts are joining. Solidification time of the identical casting poured into the resin moulding sand is presented on Fig. 1b. Here, the conical part solidified from 210 to 850 seconds and the cylinder one in the interval of 850 to 1300 seconds. The hot spot's solidification was completed after 1415 seconds. Generally, we can say that the solidification time of the casting in the resin moulding sand was approximately double compared with the bentonite one, what can be regarded as the acceptable agreement of the calculation with results of simulation. Experiments have confirmed that the casting's thinnest sections of $2 \mathrm{~mm}$ poured into the moulding sand bonded with phenol-formaldehyde resin solidified in equal time as with a section of $3 \mathrm{~mm}$ in the bentonite sand [2]. The possibility to pour castings having a modulus of 1 to 1.5 we verified successfully in series production.

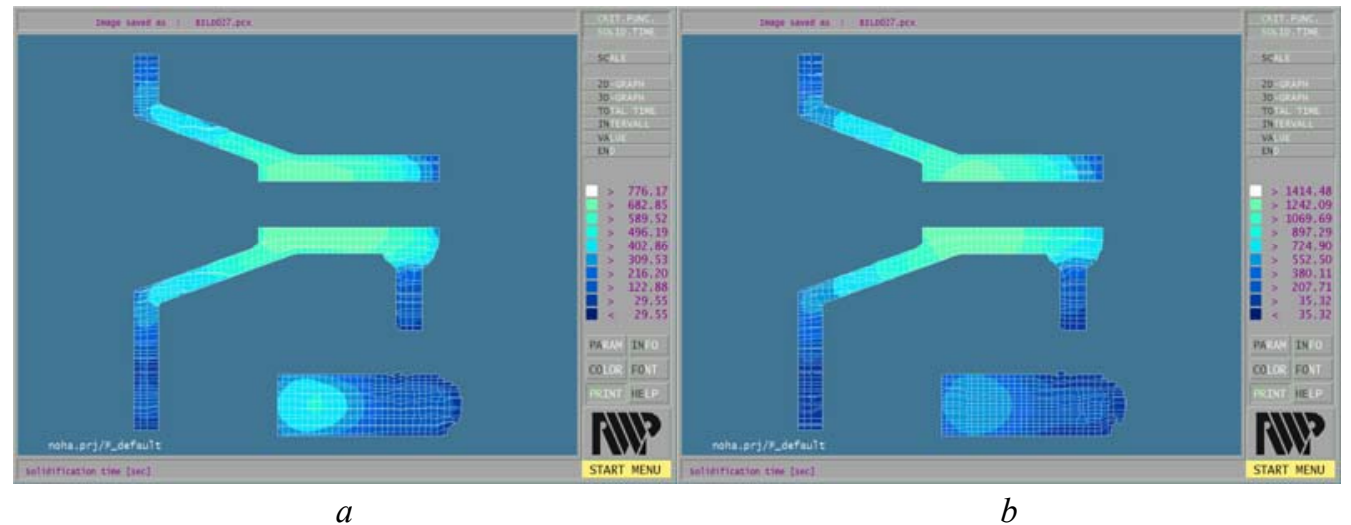

Figure 1 Solidification time of casting cooling in moulding sands: (a) bentonite, (b) resin

\section{CONCLUSION}

Currently, it is good practice to utilize the chemically bonded moulding sand to produce castings from SGI because they can bring significant qualitative benefit at a low raise in production costs. In the case of the heavy sectioned castings $(t>50 \mathrm{~mm})$, the considerably extended time of solidification causes fading of inoculation, and therefore, the inevitable worsening of quality parameters for SGI. Apart from many positive technological characteristics, the obvious benefit of applying water-free moulding sand is the fall in cooling capacity, which shifts the minimal possible thickness of the casting's wall to the range of about $2 \mathrm{~mm}$.

This work was supported by the Slovak Research and Development Agency in the frame of the bilateral Slovakia-Czech Republic cooperation, grant no. SK-CZ-0138-11.

\section{REFERENCES}


[1] Murgaš, M. - Pokusová, M. - Pokusa, A.: Určenie zloženia liatiny s gul'ôčkovým grafitom v závislosti od hrúbky stien odliatkov. In Slévárenství, 1998, roč. 46, č. 3-4, s. 101.

[2] Lekach, S.N. - Bestužev, N.I.: Vnepečnaja obrabotka vysokokačestvennych čugunov v mašinostrojeniji. Minsk: Navuka i technika, 1992.

[3] Pokusová, M. - Murgaš, M.: Using the modulus at optimizing of ductile cast iron chemical composition. In: Chvorinovs's colloquium. Ostrava: VŠB - TU Ostrava, 2009. CD

[4] Pokusová, M. - Pokusa, A. - Mojžiš, M.: Computer simulation of solidification for in-mold technology. In Acta Metallurgica Slovaca. 2007, roč. 13, č. 4, s. 76-79. 